adjusted odds ratio $(\mathrm{OR})=0.62,95 \% \mathrm{CI}$ : 0.52 to 0.74$)$ and a lower proportion of survived one year ( $43 \%$ v $27 \%$; OR=0.78; 0.68 to 0.89 ). Urgency of GP referral did not affect treatment intent or survival. Routes to diagnosis varied across the 30 cancer networks, with the proportion of patients diagnosed after emergency admission ranging from $8.7 \%$ to $32.3 \%$

Conclusion Outcomes for cancer patients are worse if diagnosed after emergency admission. Networks should examine the causes of large regional variations to reduce rates of diagnosis after emergency admission.

\section{PS38 SARCOPENIC OBESITY AND RISK OF ALL-CAUSE AND CARDIOVASCULAR MORTALITY IN OLDER MEN}

\section{doi:10.1136/jech-2012-201753.137}

${ }^{1} \mathrm{JL}$ Atkins, ${ }^{2} \mathrm{PH}$ Whincup, ' $\mathrm{RW}$ Morris, ${ }^{1} \mathrm{SG}$ Wannamethee. 'Department of Primary Care and Population Health, UCL, London, UK; ${ }^{2}$ Division of Population Health Sciences and Education, St George's University of London, London, UK

Background Sarcopenic obesity refers to the age-associated loss of muscle mass coupled with high adiposity levels. Although it is known that both obesity and low muscle mass are associated with increased mortality, few studies to date have examined the combined effects of sarcopenia and obesity on all-cause or cardiovascular mortality. We examined the relationship between sarcopenic obesity and all-cause and cardiovascular mortality in older men.

Methods 4,252 men from the British Regional Heart Study, aged 60-79 years in 1998-2000, were followed prospectively until 2011 for mortality. At baseline, midarm muscle circumference (MAMC) measurement was used to provide information on muscle mass and measurement of bioelectrical impedance analysis (BIA) to provide fat free mass index. Obesity was assessed by measurement of body mass index (BMI), waist circumference (WC), and BIA (providing fat mass, FM, index). Participants were classified as either normal, sarcopenic, obese, or sarcopenic obese using varying measures of adiposity and muscle mass, since no consensus definition for sarcopenic obesity exists. Associations between the four sarcopenic obesity groups and mortality rates (all-cause and cardiovascular) were examined using Cox regression, adjusting for age, smoking, alcohol intake, social class and physical activity.

Results There were 1,456 deaths during follow-up (mean=10.1years), 578 (40\%) of which were cardiovascular. Obesity (assessed by BMI, WC and FM index) was not significantly associated with all-cause mortality, but BMI and WC were significantly associated with cardiovascular mortality $(p<0.05)$. Low muscle mass (MAMC only) was significantly associated with all-cause mortality $(p<0.001)$ but not with cardiovascular mortality. Sarcopenic obese men (MAMC $\leq 24.95 \mathrm{~cm}$; WC $>102 \mathrm{~cm}$ ) had the greatest relative risk of all-cause mortality (RR: 1.66, 95\% CI:1.23-2.25). Risks of all-cause mortality were also increased among men who were sarcopenic only (RR: 1.25, 95\% CI:1.09-1.43) and obese only (RR:1.44, 95\% CI:1.25-1.64) compared with normal men. Similar, though weaker, relationships were seen using MAMC and BMI to define sarcopenic obesity, but not when using combined BIA measures. Sarcopenic obese men had a less marked excess cardiovascular mortality risk (RR: 1.20, 95\% CI:0.69-2.08), compared with obese men (RR: 1.35, 95\% CI:1.09-1.67).

Conclusion Sarcopenic obese older men are at an increased risk of all-cause mortality compared with those with only sarcopenia or obesity or normal body composition. MAMC and WC appeared to be the best markers of muscle mass and obesity for predicting allcause mortality, compared with BMI and BIA measures. The added effect of sarcopenia and obesity, however, did not have a synergistic effect on cardiovascular mortality. Efforts to promote healthy ageing in the elderly should focus on both preventing obesity and maintaining muscle mass.

\section{PS39 ALCOHOL AND HARM TO OTHERS IN RUSSIA: LONGITUDINAL ANALYSIS OF COUPLE DRINKING AND SUBSEQUENT DIVORCE}

doi:10.1136/jech-2012-201753.138

${ }^{1} \mathrm{~K}$ Keenan, ${ }^{2} \mathrm{E}$ Grundy, ${ }^{3} \mathrm{MG}$ Kenward, ${ }^{4} \mathrm{DA}$ Leon. ${ }^{1}$ Department of Non-communicable Disease Epidemiology, LSHTM, London, UK; '2Department of Population Studies, LSHTM, London, UK; ${ }^{3}$ Department of Medical Statistics, LSHTM, London, UK; ${ }^{4}$ Department of Non-communicable Disease Epidemiology, LSHTM, London, UK

Background In Russia male drinking patterns have serious negative health effects; however the impact of alcohol on divorce is relatively unexplored. In other settings heavy drinking and discrepant drinking within couples increases the probability of marital breakdown. Longitudinal data, rather than cross-sectional, is preferable to establish the direction of any causal link.

Methods The association between married couple drinking patterns and subsequent divorce was investigated in a national population-based panel study in Russia. Follow-up data on 4,266 married couples was extracted from 14 consecutive annual rounds (19942009) of the Russian Longitudinal Monitoring Survey. The overall follow-up rate of couples was $90 \%$, and loss to follow-up was unrelated to drinking behaviour. At interview couples provided information about family relationships, drinking habits in the last 30 days and socio-demographic variables. Discrete time hazard models were fitted using pooled logistic regression to estimate the probability of divorce among married couples as a function of the previous round's drinking patterns and other covariates.

Results Increased odds of divorce were associated with greater frequency of husband drinking $(\mathrm{P}<0.001)$ and greater frequency of wife drinking $(\mathrm{P}<0.001)$, and remained significant after mutual adjustment. Wife's hazardous drinking was also associated with a higher risk of divorce (OR 1.45, 95\% CI 1.06-1.92) after adjustment for husband's drinking. Husbands who were abstainers also had raised odds of divorce compared to moderate drinkers (OR 1.36, CI 1.011.84). There was a significant positive relationship between husband's maximum daily volume of ethanol from vodka and divorce, after adjustment for frequency. After testing for interaction between husband's and wife's drinking, there was no evidence that couples with discrepant drinking frequencies had increased risk of divorce.

Conclusion This study adds to the very sparse literature investigating the association of drinking with divorce using longitudinal data. The results suggest that in Russia heavy and frequent drinking of both husbands and wives put couples at greater risk of future divorce. The thresholds where frequency and volume adversely affect marital stability are higher in husbands, than in wives. Male abstainers have a higher degree of marital dysfunction, lending support to the idea that many Russian male abstainers are ex-drinkers. More research is needed to understand the causal pathways from drinking to marital breakdown in Russia, and the overall population-level impact of drinking on partnerships.

\begin{tabular}{l}
\hline PS40 PROFILES OF POLYDRUG USE AT A LOCAL AND A \\
NATIONAL LEVEL: RISK FACTORS AND ASSOCIATIONS \\
WITH MENTAL HEALTH AND FUNCTIONING
\end{tabular}

doi:10.1136/jech-2012-201753.139

JL Clark, S Frissa, S Hatch, M Hotopf. Psychological Medicine, Institute of Psychiatry King's College London, London, UK

Background Polydrug use is an understudied phenomenon with potential implications for individual functioning and health. Its frequency within the general public and its individual costs thus need to be documented. This study's aims were to compare the types of polydrug users in the general public at a national and a local level, and to examine how risk factors and impairments differed between them. 